\title{
Reaching across the abyss: recent advances in functional magnetic resonance imaging and their potential relevance to disorders of consciousness
}

\author{
Andrea Soddu ${ }^{1,4, *}$, Melanie Boly ${ }^{1,2}$, Yuval Nir ${ }^{5}$, Quentin Noirhomme ${ }^{1}$, \\ Audrey Vanhaudenhuyse ${ }^{1}$, Athena Demertzi ${ }^{1}$, Anat Arzi $^{3}$, Smadar Ovadia ${ }^{3}$, \\ Mario Stanziano ${ }^{4}$, Michele Papa ${ }^{4}$, Steven Laureys ${ }^{1,2}$ and Rafael Malach ${ }^{3}$ \\ ${ }^{1}$ Coma Science Group, Cyclotron Research Centre, University of Liège, Belgium \\ ${ }^{2}$ Neurology Department, CHU Sart Tilman Hospital, University of Liège, Belgium \\ ${ }^{3}$ Department of Neurobiology, Weizmann Institute of Science, Rehovot, Israel \\ ${ }^{4}$ Medicina Pubblica Clinica e Preventiva, Second University of Naples, Naples, Italy \\ ${ }^{5}$ Department of Psychiatry, University of Wisconsin, Madison, WI, USA
}

\begin{abstract}
Disorders of consciousness (DOC) raise profound scientific, clinical, ethical, and philosophical issues. Growing knowledge on fundamental principles of brain organization in healthy individuals offers new opportunities for a better understanding of residual brain function in DOCs. We here discuss new perspectives derived from a recently proposed scheme of brain organization underlying consciousness in healthy individuals. In this scheme, thalamo-cortical networks can be divided into two, often antagonistic, global systems: (i) a system of externally oriented, sensory-motor networks (the "extrinsic" system); and (ii) a system of inward-oriented networks (the "intrinsic" or default system). According to this framework, four distinct mental states would be possible that could be relevant for understanding DOCs. In normal healthy volunteers and locked-in syndrome patients, a state of high functionality of both the extrinsic and intrinsic or default systems is expected - associated with full awareness of environment and self. In this case, mental imagery tasks combined with fMRI can be used to detect covert awareness in patients that are unable to communicate. According to the framework, two complementary states of system imbalance are also possible, in which one system is in a hyperfunctional state, while the other is hypoactive. Extrinsic system hyperfunction is expected to lead to a state of total sensory-motor "absorption" or "lost self." In contrast, intrinsic or default system hyperfunction is expected to lead to a state of complete detachment from the external world. A state where both extrinsic and intrinsic systems are hypofunctional is predicted to lead to markedly impaired consciousness as seen in DOCs. Finally, we review the potential use of ultra-slow fluctuations in BOLD signal as a tool for assessing the functional integrity of extrinsic and intrinsic systems during "resting state" fMRI acquisitions. In particular, we
\end{abstract}

\footnotetext{
*Corresponding author.

Tel.: +32 436623 35; Fax: +3243662946;

E-mail: Andrea.Soddu@ulg.ac.be
} 
discuss the potential provided by assessment of these slow spontaneous BOLD fluctuations as a novel tool in assessing the cognitive state and chances of recovery from brain pathologies underlying DOCs.

Keywords: coma; consciousness; resting state; spontaneous activity; imagery; functional magnetic resonance imaging; default network

\section{Introduction}

Disorders of consciousness (DOC) are a devastating spectrum of clinical conditions involving profound disruption in global conscious states due to massive brain lesions (Bernat, 2006; Giacino et al., 2002; Laureys et al., 2004; Plum and Posner, 1972; Schiff, 2006b). Clinical characterization of the different DOCs is based on two main distinct components of human consciousness: arousal and awareness (Plum and Posner, 1972). If arousal refers to the behavioral alternation of sleep and wakefulness, awareness refers to the collective thoughts and feelings of an individual (Laureys, 2005). Coma is characterized by the absence of arousal and hence of awareness. Vegetative-state patients are aroused but unaware of environment and self (Jennett and Plum, 1972). Minimally conscious state patients are unable to reliably communicate but show reproducible behavioral evidence of awareness of environment or self (Giacino et al., 2002, 2004; Majerus et al., 2005). Locked-in syndrome patients (Plum and Posner, 1972) are fully conscious but have no means of producing speech, limb, or facial movements, except for small movements of the eyes or eyelids.

While progress has been made in describing DOCs from the clinical perspective (Giacino et al., 2004), we focus in the present review on examining DOCs from the point of view of recent developments and understandings derived from the healthy human brain. We argue that recent insights obtained through functional magnetic resonance imaging (fMRI) are relevant to a better understanding of DOCs and have the potential of allowing a better diagnosis and treatment (Giacino et al., 2006; Laureys et al., 2006; Schiff, 2006a, b). Specifically, we will focus on assessing brain function in a set of areas, termed the "default" network (Raichle et al., 2001; Raichle and Snyder, 2007), characterized by higher activity at rest than during externally oriented sensorymotor or cognitive tasks. This network appears to show antagonistic behavior to sensory-motor areas, which show increased fMRI signal under such tasks. Importantly, areas belonging to the default network also show a tendency for coherent fluctuations both during rest (Greicius et al., 2003; Nir et al., 2006) and during visual activation (Hasson et al., 2004) further supporting their association within a common functional system.

Based on the functional antagonistic profile of the sensory-motor cortex on the one hand and the default system on the other, as well as their complementary neuroanatomical organization (Boly et al., 2007a; Fox et al., 2005, 2009; Golland et al., 2007, 2008; Tian et al., 2007; see Fig. 1) we have proposed (Boly et al., 2008a, b; Golland et al., 2008) a fundamental "dual" subdivision of the human cortex into two basic - "extrinsic" versus "intrinsic" - functionalities. More specifically, we hypothesize that the cerebral cortex can be subdivided according to two basic functional orientations - an "extrinsic" orientation, which engages sensory-motor cortices, and an "intrinsic" orientation, which engages the default system. Sensory-motor cortices are involved in the processing of information immediately incoming from the external world, while the complementary "intrinsic" or default system appears to be involved in self-representations, episodic memory, mind wandering, and stimulus-independent thoughts (e.g., see Goldberg et al., 2006; Mason et al., 2007; D'Argembeau et al., 2005; Laureys et al., 2007).

We here will address the study of residual brain function in DOCs from the perspective of this "extrinsic" versus "intrinsic" functional subdivision and propose a conceptual framework around which to organize our knowledge and hypotheses concerning DOCs. Specifically we will consider 


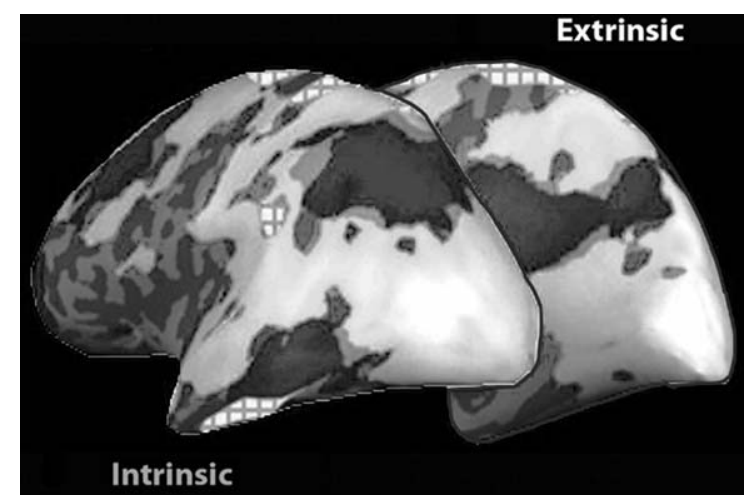

Fig. 1. An illustration of the proposed fundamental specialization of the human cortex showing the "extrinsic" sensory motor system and the "intrinsic" or default networks. It is hypothesized that the extrinsic system specializes in processing incoming information from the external environment, while the more enigmatic intrinsic or default system is specialized in internally oriented functions. Note the complementary nature of the "extrinsic" and "intrinsic" or default networks. Adapted from Golland et al. (2007).

four different mental states associated with different extrinsic-intrinsic organizations, as predicted by our framework.

\section{High extrinsic and intrinsic functionality: the "locked-in" syndrome}

The first case to consider is the situation in which both the extrinsic and intrinsic systems are highly functional yet the patient is unable to communicate or report in an effective manner about her or his mental state. In the case of locked-in syndrome this situation of disconnection between internal states and verbal or nonverbal report is due to quadriplegia and anarthria, classically caused by a ventral pontine lesion, disrupting the corticospinal and corticobulbar pathways (Plum and Posner, 1972; Smart et al., 2008). In the case of fully conscious but completely paralyzed patients (i.e., complete locked-in syndrome; Schnakers et al., 2009), fMRI may be able to detect response to command using brain activation in the absence of any overt motor response. fMRI activation paradigms offer the opportunity to directly communicate with locked-in syndrome patients by assessing responses from their brains without dependence on motor output (Birbaumer et al., 2008, 2006; Sorger et al., 2009). A demonstration that this method could effectively work was provided in recent years by the successful use of mental imagery tasks and fMRI imaging to uncover awareness in a patient clinically assessed as vegetative (Boly et al., 2007b; Owen et al., 2006). Two important methodological considerations have to be taken into account while developing fMRI activation paradigms. First, we need to find tasks that produce the most reliable and robust activation pattern in a single subject. Second, we need to be cautious in designing the fMRI paradigms, in order to avoid brain activations which could occur passively, in the absence of any willful mental effort. To address these two issues, we conducted two fMRI experiments in healthy individuals.

In the first experiment, six healthy subjects were scanned in a $3 \mathrm{~T}$ MRI scanner during: (i) passive listening to verbal commands and (ii) active mental imagery in response to the same instructions. Mental imagery tasks included: (1) imagining opening and closing the left hand, (2) mental calculation (counting down), (3) imaging preparing luggage, (4) imaging walking from home to work, (5) covertly describing a face, (6) imaging filling in a check, and (7) covertly describing ones own thoughts. An eighth condition of rest (eyes closed) was used as a baseline. In the second experiment, we compared visual mental imagery (eyes closed) with passive visual stimulation (Farah, 1989; Ganis et al., 2004). Here, eight healthy subjects were 
scanned in four different conditions: (i) visual, (ii) imagery, (iii) visual and verbal description (covert), and (iv) imagery and verbal description (covert). The tasks (lasting 12s) included viewing or imaging: (a) walking from home to work, (b) filling a check, (c) observing the own face, and (d) preparing a luggage. A fifth condition of rest (blank screen for visual and keeping the eyes closed for imagery) was used as reference "baseline" activity. A common finding for all active tasks in the first experiment (Fig. 2) was a widespread activation encompassing bilateral intraparietal sulcus, primary sensory-motor areas, supplementary motor area, parahippocampal gyrus, inferior and middle temporal gyri, language-related and inferior frontal areas. Together with the activation pattern, there was also a consistent activity reduction in the intrinsic or default network during performance of the tasks. In Fig. 2, the superimposed contour map show the intrinsic or default network (identified using independent component analysis from a group of seven healthy volunteers scanned during $10 \mathrm{~min}$ "eyes-closed resting state") encompassing precuneus and adjacent posterior cingulate cortex, mesiofrontal cortex and adjacent anterior cingulate cortex, and bilateral temporoparietal junctions areas. The combined increased activation of the extrinsic network and the deactivation of the intrinsic system suggest that mental imagery tasks have a preferentially extrinsic component, likely due to a process of "replay" of extrinsic sensorymotor activations (Gelbard-Sagiv et al., 2008). These activations were consistent across individual subjects. Table 1 shows identified areas for the seven different mental imagery tasks. In line with previous studies (e.g., Boly et al., 2007b), the spatial navigation task (imagining walking from home to work) was among the tasks showing the most consistent activity across subjects (together with imagery tasks of writing a check and preparing a luggage). Figure 3 shows the activation patterns during two different sessions for the active mental imagery task (spatial navigation) compared to activation induced by passive listening to the same task instructions. While passive listening did not elicit activation, both active imagery tasks elicited activation of parahippocampal areas, well known to be involved in spatial navigation tasks (Epstein et al., 1999; Epstein and Kanwisher, 1998). This differential activation in active

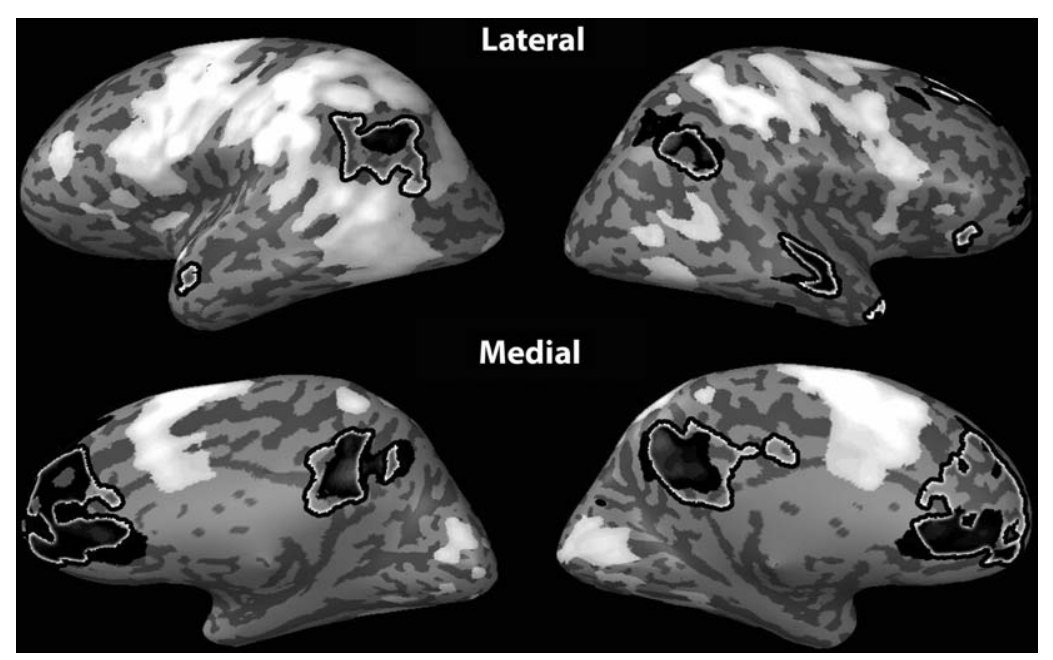

Fig. 2. Common activation and deactivation patterns (as compared to eyes-closed resting) observed during seven mental imagery tasks (i.e., imagining opening and closing the left hand; mental calculation; imaging preparing luggage; imaging walking from home to work; covertly describing ones own face; imaging filling in a check and covertly describing ones own thoughts). Fixed effects group analysis thresholded at false discovery rate corrected $p<0.05$. The contours of the independently identified default mode network are contoured in black. Note the robust imagery-related activation overlapping with regions associated with the extrinsic system (see text for details). 
Table 1. Random effect group analyses identifying areas activating during the seven mental imagery tasks as compared to eyesclosed resting state

\begin{tabular}{|c|c|c|c|c|c|c|}
\hline Imagery task & Region & $X$ & $Y$ & $Z$ & $T$-value & $p$-value \\
\hline Imagining opening and & Superior parietal lobe & -36 & -35 & 40 & 9.3 & $<0.001$ \\
\hline \multirow[t]{2}{*}{ closing the left hand } & Supplementary motor area & 2 & 7 & 50 & 6.5 & $<0.001$ \\
\hline & Middle temporal gyrus & -50 & -52 & 3 & 6.4 & $<0.001$ \\
\hline \multirow[t]{3}{*}{ Counting down } & Superior parietal lobe & -42 & -33 & 40 & 5.7 & $<0.001$ \\
\hline & Precentral gyrus & -53 & 2 & 23 & 5.5 & $<0.001$ \\
\hline & Supplementary motor area & -1 & 4 & 51 & 5.2 & $<0.001$ \\
\hline \multirow{3}{*}{ Imaging preparing luggage } & Superior parietal lobe & -35 & -34 & 42 & 6.2 & $<0.001$ \\
\hline & Parieto-occipital sulcus & -18 & -59 & 44 & 5.4 & $<0.001$ \\
\hline & Supplementary motor area & -5 & 5 & 48 & 5.3 & $<0.001$ \\
\hline \multirow{3}{*}{ Imaging walking from home to work } & Parahippocampal gyrus & -13 & -56 & 10 & 7.1 & $<0.001$ \\
\hline & Superior parietal lobe & -35 & -34 & 38 & 6.6 & $<0.001$ \\
\hline & Medial occipitotemporal gyrus & -24 & -36 & -7 & 5.7 & $<0.001$ \\
\hline \multirow{3}{*}{ Describing a face } & Superior parietal lobe & -44 & -30 & 38 & 7.9 & $<0.001$ \\
\hline & Supplementary motor area & -5 & 5 & 50 & 5.9 & $<0.001$ \\
\hline & Lateral occipitotemporal gyrus & -40 & -54 & -14 & 5.3 & $<0.001$ \\
\hline \multirow[t]{3}{*}{ Imaging filling a check } & Superior parietal lobe & -48 & -29 & 42 & 6.7 & $<0.001$ \\
\hline & Superior parietal lobe & -33 & -35 & 40 & 6.4 & $<0.001$ \\
\hline & Middle temporal gyrus & -44 & -45 & -4 & 6.2 & $<0.001$ \\
\hline \multirow[t]{3}{*}{ Describing own thoughts } & Middle frontal gyrus & -44 & -1 & 51 & 4.9 & $<0.001$ \\
\hline & Superior parietal lobe & -46 & -33 & 41 & 4.6 & $<0.001$ \\
\hline & Superior temporal gyrus & -61 & -39 & 10 & 4.3 & $<0.001$ \\
\hline
\end{tabular}

compared to passive conditions strengthens the hypothesis that observed brain responses are indeed associated with intentional mental effort and are not merely induced by passive listening to the task instructions. Finally, Fig. 4 illustrates brain activation patterns when one sees a video of oneself walking (visual) compared to imagining oneself walking, and when the visual imagery process is accompanied by a covert description. The activation of Broca's area both in the "imagining" and "imagining and verbal description" conditions suggests that even when subjects were asked to perform the mental imagery tasks using only visualization strategies they could not avoid adding a verbal component to the visual imagery aspect.

Overall, three conclusions can be drawn from these results. First, robust brain activations can be elicited and measured with fMRI without the subjects performing any overt responses. This brain activation seems particularly robust in tasks involving action planning, while recognition tasks such as imagining faces seemed less effective. Second, the obtained activations could be differentiated in a reproducible manner from the passive conditions in which subjects did not engage in any active mental imagery. Finally, requiring a verbal description of the cognitive action while performing mental imaging tasks increases the neural activation intensity and extent. These results are encouraging in showing that a consistently detected increase in fMRI signal can be obtained when subjects are engaged in active mental imagery, and that this activation pattern can be reliably differentiated from the more automatic neural responses to presentation of the task instructions. On the other hand, for communication purposes, it appears that relying only on the patterns of activations associated with different mental imagery tasks may not be an effective solution as in the above-reported study many patterns of activations were rather similar between tasks. In this context, it is useful to also collect information on the timing of the fMRI response to a given task (i.e., starting time and latency of the subject's BOLD response) in order to better segregate the activation patterns elicited by different mental imagery tasks (see Sorger et al., 2009). 


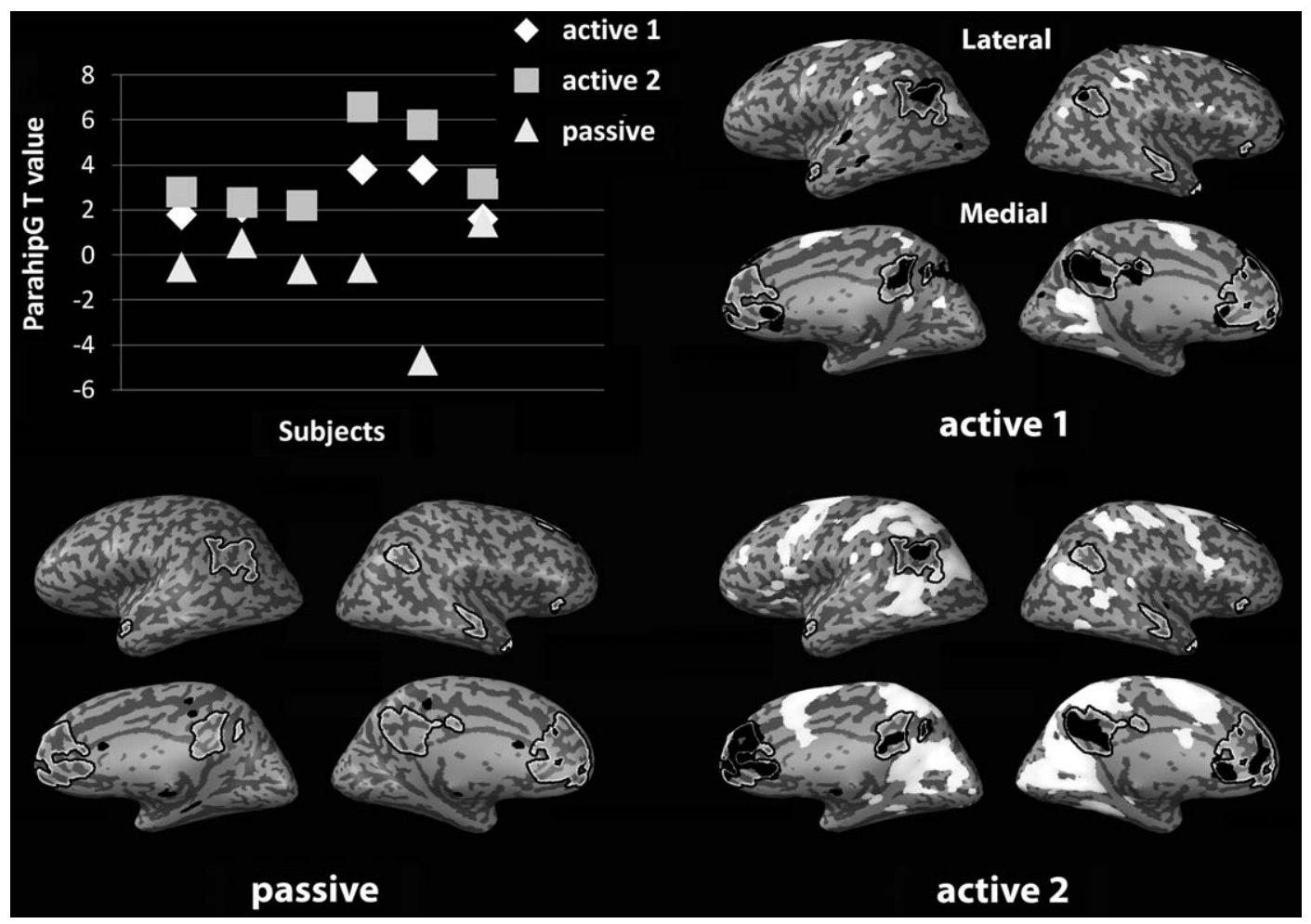

Fig. 3. Activation patterns observed during two different sessions of active mental imagery (spatial navigation) compared to activation induced by passive listening to the same task instructions. Fixed effects group analysis thresholded at false discovery rate corrected $p<0.05$. Superimposed are the contours of the default mode network as in Fig. 2. Parahippocampal gyrus activity (Talairach coordinates $X=-13, Y=-15, Z=10 \mathrm{~mm}$ ) is shown for each of the six healthy volunteers for the three acquisitions. Note the consistently higher activation during active imagery compared to passive listening.

We described here the first condition predicted by the framework, that is, full consciousness with preserved functionality of both extrinsic and intrinsic networks, and the use of mental imagery tasks to detect this condition in brain-damaged patients. We will now consider two hypothetical situations in which there is an imbalance between the extrinsic and intrinsic systems.

\section{"Losing the self": hypoactivity of the intrinsic system}

Perhaps the most robust fMRI finding which concerns the functionality of the intrinsic or default system is the consistent reduction of activity in this network during performance of cognitively demanding externally oriented tasks such as visual recognition and motor planning (Gusnard et al., 2001). In contrast, the intrinsic system shows increased fMRI activity during notask "resting" conditions, leading to the notion that this network might be a "task-negative" system (Fox et al., 2005, 2009) or be engaged in a "default" function during resting conditions (Raichle et al., 2001; Raichle and Snyder, 2007). It has been proposed that this reduced activity may be attributed to the fact that during an intense externally oriented task, the performing subject is fully attentive to the external world, and metaphorically speaking "loses itself" in the act (Goldberg et al., 2006; Golland et al., 2007). What then could be the outcome of a permanent reduction in the intrinsic system activity due to brain abnormality? Since no direct data are available, yet from DOCs one can only 


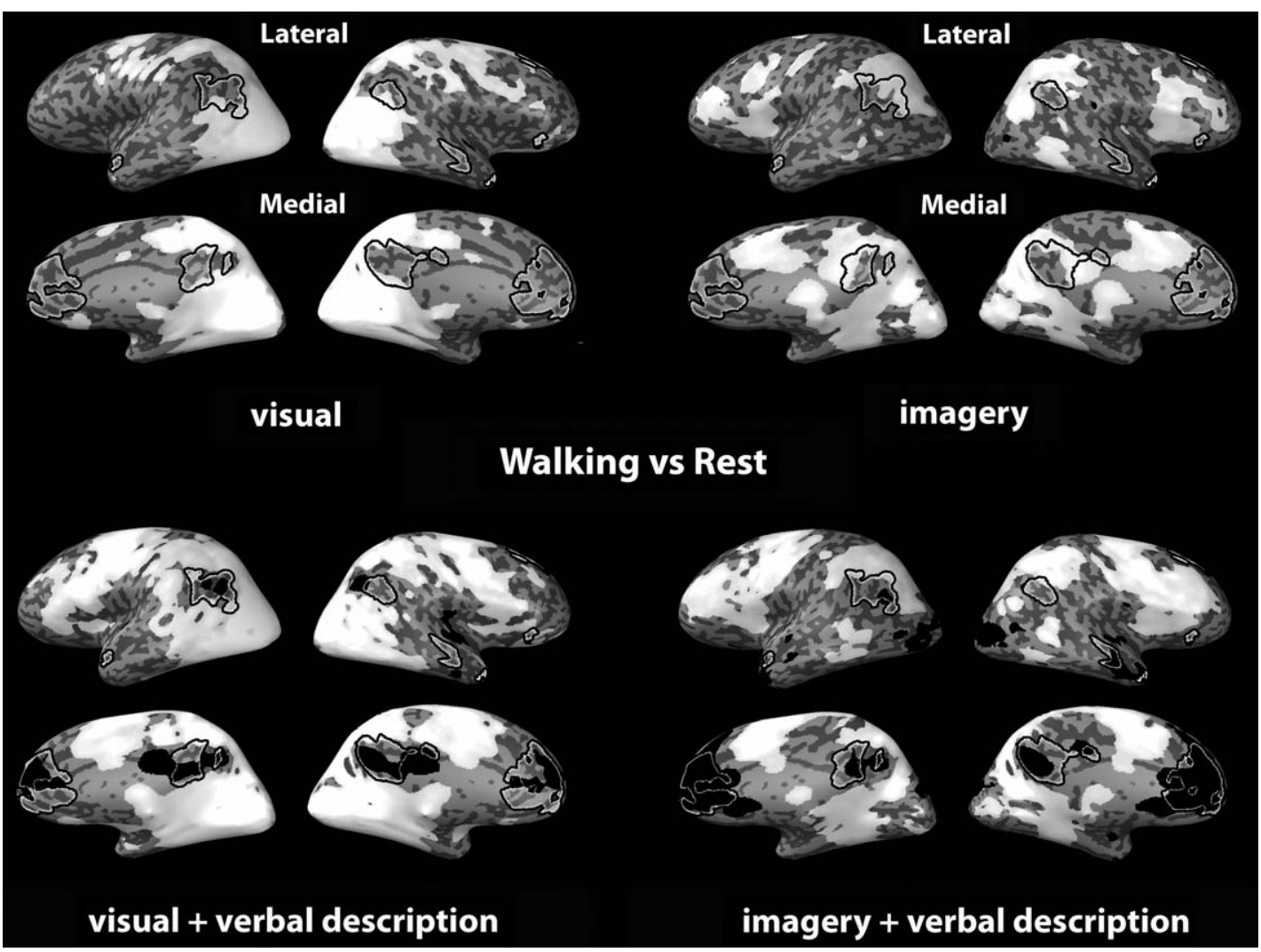

Fig. 4. Activation patterns induced by passively seeing a video of oneself walking (visual); imagining oneself walking (imagery), covert verbal description during the visual stimulation, and covert verbal description during the mental visual imagery task. Fixed effects group analysis thresholded at false discovery rate corrected $p<0.01$. Superimposed are the contours of the default mode network. Note the substantial overlap of activity patterns between all four conditions, and the enhancement in neural activation associated with verbal descriptions.

extrapolate from studies in healthy individuals. It appears that some aspect of the intrinsic system function may be associated with voluntary decisions and action initiation (Goldberg et al., 2008). Extrapolating from such findings leads to the conjecture that a possible consequence of a pathological damage to this system might be a condition akin to catatonic or akinetic mutism (Naccache et al., 2004) in which the patient is unable to voluntarily initiate a motor action - in De Tiege et al. (2003) akinetic mutism was indeed linked to medial prefrontal dysfunction. Note that such a condition, associated with lack of movement, could in principle masquerade as a DOC since the patient may not initiate any response or communication.

\section{"Self-centered absorption"}

The third condition predicted by the framework, in which the extrinsic system is hypofunctional, would be reflected by the disengagement of the subjects from the external environment. Few hints associate such conditions with "mind wandering," where typically high default network activity is observed (Christoff et al., 2009; Gilbert et al., 2007; Mason et al., 2007; Wang et al., 2009). While these notions fit the conceptual framework of an antagonistic relationship between the extrinsic and intrinsic networks - that is, an enhancement in intrinsic activity comes at the expense of processing of the extrinsic information, the relevant data are too scant as yet. Nevertheless, 
it is tempting to speculate how a pathological imbalance in which the extrinsic system is largely inactive should be reflected in the mental state of DOCs. From our conceptual framework we anticipate that such an imbalance would manifest itself again in a severe motor inaction, but also in a reduced sensory responsivity (since the patient is detached and self-absorbed and is incapable of orienting to the external world) either in terms of receiving sensory signals, or emitting motor actions. Behaviorally, then, the two conditions ("lost self" and "self-centered absorption") could paradoxically lead to similar behavioral manifestations. Again, brain imaging, and particularly the study of spontaneous cerebral BOLD fluctuations by means of fMRI, might provide useful clues in diagnosing these hypothetical conditions - as will be discussed later. Finally, we consider the possibility that both systems are abnormally hypofunctional. In this case, we predict a deep DOC in which the patient does not respond and is also incapable of initiating any voluntary communication. In such severe and widespread brain abnormalities we expect a greatly reduced brain metabolism and a general reduction in neuronal activity.

\section{Spontaneous fMRI activity patterns as a diagnostic tool in DOCs}

Although the above discussions of possible abnormalities associated with the new framework of brain organization are largely hypothetical and speculative, they do illustrate the complexity of brain abnormalities that could produce behavioral symptoms which may deceptively appear identical at the behavioral level. Here, the potentially powerful approach of functional brain imaging (Hirsch, 2005; Laureys et al., 2000, 1999a, b; Schiff et al., 2005) and particularly of fMRI may come as a useful and incisive tool. It may be argued that functional neuroimaging, if using active paradigms (Boly et al., 2007b; Owen et al., 2006, 2007) will be useful only in those limited "pseudo locked-in" cases where the patient behaviorally looks unconscious but in reality is fully aware and can initiate complex voluntary mental activity. Recent progress in studying spontaneous brain activity (Biswal et al., 1995; Cordes et al., 2000; Damoiseaux et al., 2006; Fox and Raichle, 2007; Fox et al., 2005; Greicius et al., 2003; Lowe et al., 1998; Mitra et al., 1997; Nir et al., 2006; Vincent et al., 2007; Xiong et al., 1999) demonstrating activity patterns that emerge without any task or sensory stimulation, promise for studying higher-order associative network functionality and revealing their potential abnormalities in the absence of the patients' collaboration (Boly et al., 2009b; Greicius et al., 2004; Rombouts et al., 2009).

The functional significance of low-frequency fMRI activity fluctuations remains yet poorly understood. A demonstration that such spontaneous activity occurs in primary sensory systems is of particular importance in this context. Indeed, the spontaneous nature of brain activity can be ascertained in such systems, if the sensory stimuli are completely blocked, and careful controls for imagery and attention are used (Nir et al., 2006, 2008). Recent research using intracranial recordings have revealed a putative electrophysiological correlate of such fMRI spontaneous activity in the neuronal responses of human cortex (He et al., 2008; Nir et al., 2008). More specifically, during resting-state conditions the human cortex manifests ultra-slow modulations of neuronal activity reflected both in firing rate modulations of individually isolated cortical neurons, as well as in modulation of high-frequency gamma power of local field potentials. These ultra-slow fluctuations show a remarkable coherence across functionally similar sites, and interestingly, are greatly accentuated during different sleep stages. Figure 5 depicts an example of such spontaneous activity recorded bilaterally from human auditory cortex during quiet rest (stage II sleep), showing a remarkable coherence of the activity in auditory cortices of both hemispheres. Although these activity patterns have a much slower dynamics than task-related activations (Nir et al., 2008), their widespread nature and remarkable reproducibility among subjects (Damoiseaux et al., 2006) makes them a potential tool for assessing the viability and functionality of cortical networks. 


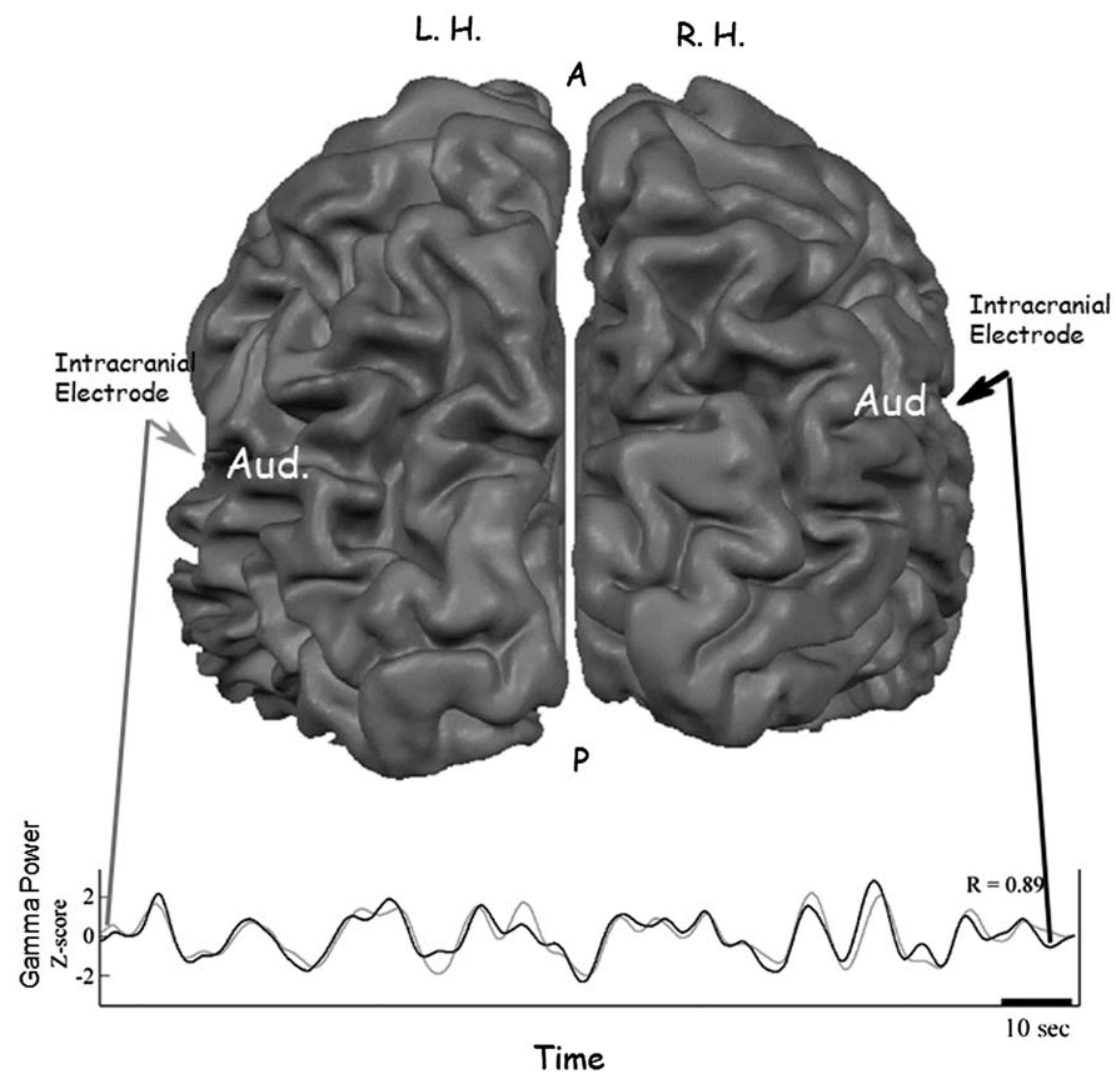

Fig. 5. Intracranial recordings obtained from bilateral auditory cortex depicting gamma power modulations in the local field potentials. Note the ultra-slow, spontaneous fluctuations and their remarkable correlation across the two hemispheres. Adapted from Nir et al. (2008).

Data-driven approaches such as independent component analysis (Hyvärinen, 1999) or $k$-clustering (Golland et al., 2008) applied to spontaneous activity, could reveal a full set of independent networks with a particular spatial distribution and a characteristic frequency power spectrum (Beckmann et al., 2005; De Luca et al., 2006; Esposito et al., 2008, 2006, 2005; Mantini et al., 2007; McKeown et al., 1998; Perlbarg and Marrelec, 2008). The advantage of studying these activity patterns is that they nicely correspond to the functional organization of global brain systems (Bullmore and Sporns, 2009; Hagmann et al., 2008; Honey et al., 2009). Thus, cortical systems, which are functionally coupled during task performance, also show a similar coupling of spontaneous activity. Consequently, these spontaneous activations offer a tool in assessing cortical functional abnormalities in patients that cannot cooperate. Indeed, a recent report has presented important evidence that such spontaneous activity can provide a sensitive marker for detecting cortical abnormalities in neurodegenerative disorders (Seeley et al., 2009). More specific to DOCs, we have recently demonstrated that default network connectivity was decreased in severely braindamaged patients in proportion to their degree of consciousness impairment Boly et al., (2009); Vanhaudenhuyse et al. (submitted), Boly et al., (2009), demonstrated absent cortico-thalamic BOLD functional connectivity (i.e., cross-correlation between precuneal areas and medial thalamus) but partially preserved cortico-cortical connectivity within the default network in a vegetative-state patient studied 2.5 years following cardio-respiratory arrest (see Fig. 6). In a more comprehensive 


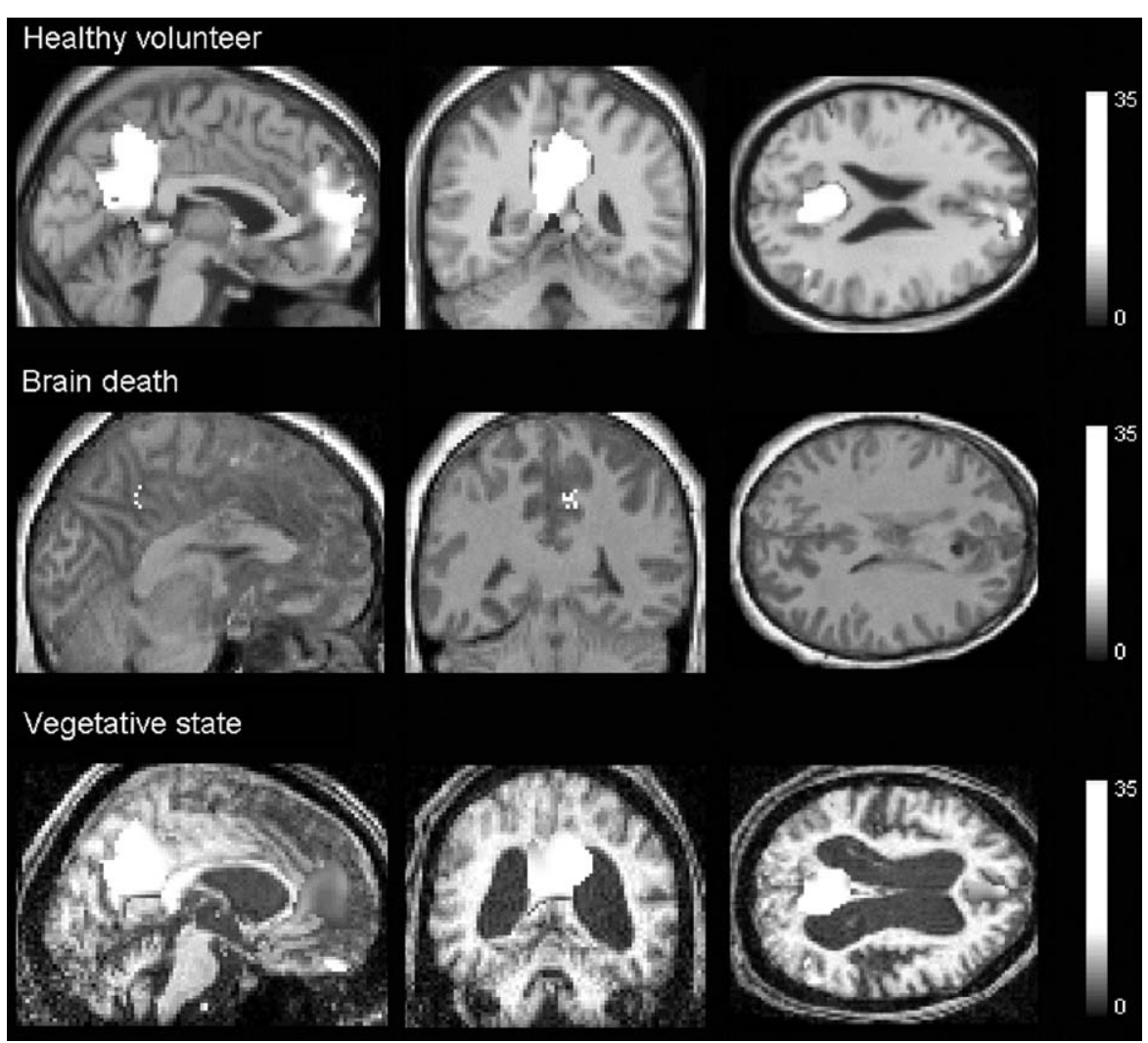

Fig. 6. Positive correlations with precuneal and posterior cingulate activity in a healthy volunteer, a patient in brain death and a patient in a vegetative state. Results are thresholded at false discovery rate corrected $p<0.001$. Note that there is no residual longrange functional connectivity in brain death. In the vegetative state, despite the presence of residual functional connectivity within the default network, the observed activity is reduced compared to healthy controls. Adapted from Boly et al. (2009).

study (Vanhaudenhuyse et al. (submitted)) 14 noncommunicative brain-damaged patients and 14 healthy controls participated in a resting-state fMRI protocol. Connectivity was investigated using probabilistic-independent component analysis and an automated template-matching component selection approach. Connectivity in all default network areas was found to be linearly correlated with the degree of consciousness, ranging from healthy volunteers and locked-in syndrome to minimally conscious, vegetative, and comatose patients. Furthermore, precuneus connectivity was found to be significantly stronger in minimally conscious patients compared to vegetative-state patients. Locked-in syndrome patients' default network connectivity was shown not to be significantly different from healthy control subjects.
A remaining issue in the study of spontaneous BOLD signal fluctuations, especially for patients that show a significantly reduced neuronal activity, is the possible contamination by noise sources (Birn et al., 2006; Chuang and Chen, 2001; Cordes et al., 2000). Different strategies have been adopted based on two major defining characteristics of spontaneous brain activity as reported also by fMRI studies: (i) their tendency to be correlated across hemispheres (Biswal et al., 1995; Cordes et al., 2000; Damoiseaux et al., 2006; Fox and Raichle, 2007; Fox et al., 2005; Golland et al., 2007; Greicius et al., 2003; Lowe et al., 1998; Nir et al., 2006; Vincent et al., 2007; Xiong et al., 1999) and (ii) their neuroanatomical selectivity, that is, such fluctuations are not global, and distinct functional systems are often decorrelated (Biswal 
et al., 1995; Cordes et al., 2000; Damoiseaux et al., 2006; Fox and Raichle, 2007; Fox et al., 2005; Golland et al., 2007; Greicius et al., 2003; Lowe et al., 1998; Nir et al., 2006; Vincent et al., 2007; Xiong et al., 1999). Data-driven approaches like independent component analysis offer the advantage to better isolate physiological artifacts from the neuronal components and are now being commonly adopted in this field (Beall and Lowe, 2007; Birn et al., 2008; Perlbarg et al., 2007).

In conclusion, the integration and cross-referencing from recent advances in studying the healthy human brain provide new conceptual frameworks and methodological approaches that could help better diagnosing and understanding DOC. We here emphasized two perspectives for such integration: (i) from a neuroanatomical point of view (i.e., the subdivision of the human cortex according to a fundamental extrinsic versus intrinsic specialization reflected in two global and complementary cortico-thalamic systems); and (ii) from a functional point of view (i.e., the discovery of spontaneous ultra-slow and coherent activity patterns). Both perspectives are likely to provide important advances in our attempts to reach across the abyss and gain further insight in the neural correlates of human consciousness.

\section{Acknowledgments}

This work was supported by ISF 160/07 and Minerva grants (RM), MIUR-Progetto FIRB Internazionalizzazione-RBIN04KW43 (AS), the European Commission (DISCOS, Mindbridge and COST), the McDonnell Foundation, the Mind Science Foundation, the Reine Elisabeth Medical Foundation, and University of Liège. M. Boly and S. Laureys are, respectively, Research Fellow and Senior Research Associate at the Belgian National Fund for Scientific Research.

\section{References}

Beall, E. B., \& Lowe, M. J. (2007). Isolating physiologic noise sources with independently determined spatial measures. Neuroimage, 37, 1286-1300.
Beckmann, C. F., DeLuca, M., Devlin, J. T., \& Smith, S. M. (2005). Investigations into resting-state connectivity using independent component analysis. Philosophical Transactions of the Royal Society of London. Series B, Biological Sciences, 360, 1001-1013.

Bernat, J. L. (2006). Chronic disorders of consciousness. Lancet, 367, 1181-1192.

Birbaumer, N., Murguialday, A. R., \& Cohen, L. (2008). Braincomputer interface in paralysis. Current Opinion in Neurology, 21, 634-638.

Birbaumer, N., Weber, C., Neuper, C., Buch, E., Haapen, K., \& Cohen, L. (2006). Physiological regulation of thinking: Brain-computer interface (BCI) research. Progress in Brain Research, 159, 369-391.

Birn, R. M., Diamond, J. B., Smith, M. A., \& Bandettini, P. A. (2006). Separating respiratory-variation-related fluctuations from neuronal-activity-related fluctuations in fMRI. Neuroimage, 31, 1536-1548.

Birn, R. M., Murphy, K., \& Bandettini, P. A. (2008). The effect of respiration variations on independent component analysis results of resting state functional connectivity. Human Brain Mapping, 29, 740-750.

Biswal, B., Yetkin, F. Z., Haughton, V. M., \& Hyde, J. S. (1995). Functional connectivity in the motor cortex of resting human brain using echo-planar MRI. Magnetic Resonance in Medicine, 34, 537-541.

Boly, M., Balteau, E., Schnakers, C., Degueldre, C., Moonen, G., Luxen, A., et al. (2007a). Baseline brain activity fluctuations predict somatosensory perception in humans. Proceedings of the National Academy of Sciences of the United States of America, 104, 12187-12192.

Boly, M., Coleman, M. R., Davis, M. H., Hampshire, A., Bor, D., Moonen, G., et al. (2007b). When thoughts become action: An fMRI paradigm to study volitional brain activity in non-communicative brain injured patients. Neuroimage, 36, 979-992.

Boly, M., Phillips, C., Balteau, E., Schnakers, C., Degueldre, C., Moonen, G., et al. (2008a). Consciousness and cerebral baseline activity fluctuations. Human Brain Mapping, 29, 868-874.

Boly, M., Phillips, C., Tshibanda, L., Vanhaudenhuyse, A., Schabus, M., Dang-Vu, T. T., et al. (2008b). Intrinsic brain activity in altered stated of consciousness-How conscious is the default mode of brain function? Annals of the New York Academy of Sciences, 1129, 119-129.

Boly, M., Tshibanda, L., Vanhaudenhuyse, A., Noirhomme, Q., Schnakers, C., Ledoux, D., et al. (2009). Functional connectivity in the default network during resting state is preserved in a vegetative but not in a brain dead patient. Human Brain Mapping 30(8), 2393-2400.

Bullmore, E., \& Sporns, O. (2009). Complex brain networks: Graph theoretical analysis of structural and functional systems. Nature Reviews. Neuroscience, 10, 186-198.

Christoff, K., Gordon, A. M., Smallwood, J., Smith, R., Schooler, J. W. (2009). Experience sampling during fMRI reveals default network and executive system contributions to mind wandering. Proceedings of the National Academy of 
Sciences of the United States of America. doi:10.1073/ pnas.0900234106.

Chuang, K. H., \& Chen, J. H. (2001). IMPACT: Image-based physiological artifacts estimation and correction technique for functional MRI. Magnetic Resonance in Medicine, 46, 344-353.

Cordes, D., Haughton, V. M., Arfanakis, K., Wendt, G. J., Turski, P. A., Moritz, C. H., et al. (2000). Mapping functionally related regions of brain with functional connectivity MR imaging. American Journal of Neuroradiology, 21, 1636-1644.

Damoiseaux, J. S., Rombouts, S. A., Barkhof, F., Scheltens, P., Stam, C. J., Smith, S. M., et al. (2006). Consistent restingstate networks across healthy subjects. Proceedings of the National Academy of Sciences of the United States of America, 103, 13848-13853.

D'Argembeau, A., Collette, F., Van der Linden, M., Laureys, S., Del Fiore, G., Degueldre, C., et al. (2005). Self-referential reflective activity and its relationship with rest: A PET study. Neuroimage, 25, 616-624.

De Luca, M., Beckmann, C. F., De Stefano, N., Matthews, P. M., \& Smith, S. M. (2006). fMRI resting state networks define distinct modes of long-distance interactions in the human brain. Neuroimage, 29, 1359-1367.

De Tiege, X., Bier, J. C., Massat, I., Laureys, S., Lotstra, F., Berre, J., et al. (2003). Regional cerebral glucose metabolism in akinetic catatonia and after remission. Journal of Neurology, Neurosurgery, and Psychiatry, 74, 1003-1004.

Epstein, R., Harris, A., Stanley, D., \& Kanwisher, N. (1999). The parahippocampal place area: Recognition, navigation, or encoding? Neuron, 23, 115-125.

Epstein, R., \& Kanwisher, N. (1998). A cortical representation of the local visual environment. Nature, 392, 598-601.

Esposito, F., Aragri, A., Pesaresi, I., Cirillo, S., Tedeschi, G., Marciano, E., et al. (2008). Independent component model of the default-mode brain function: Combining individuallevel and population-level analyses in resting-state fMRI. Journal of Magnetic Resonance Imaging, 26, 905-913.

Esposito, F., Bertolino, A., Scarabino, T., Latorre, V., Blasi, G., Popolizio, T., et al. (2006). Independent component model of the default-mode brain function: Assessing the impact of active thinking. Brain Research Bulletin, 70, 263-269.

Esposito, F., Scarabino, T., Hyvarinen, A., Himberg, J., Formisano, E., Comani, S., et al. (2005). Independent component analysis of fMRI group studies by self-organizing clustering. Neuroimage, 25, 193-205.

Farah, M. J. (1989). The neural basis of mental imagery. Trends in Neurosciences, 12, 395-399.

Fox, M. D., \& Raichle, M. E. (2007). Spontaneous fluctuations in brain activity observed with functional magnetic resonance imaging. Nature Reviews Neuroscience, 8, 700-711.

Fox, M. D., Snyder, A. Z., Vincent, J. L., Corbetta, M., Van Essen, D. C., \& Raichle, M. E. (2005). The human brain is intrinsically organized into dynamic, anticorrelated functional networks. Proceedings of the National Academy of Sciences of the United States of America, 102, 9673-9678.
Fox, M. D., Zhang, D., Snyder, A. Z., \& Raichle, M. E. (2009). The Global Signal and Observed Anticorrelated Resting State Brain Networks. Journal of Neurophysiology, 101, 3270-3283.

Ganis, G., Thompson, W. L., \& Kosslyn, S. M. (2004). Brain areas underlying visual mental imagery and visual perception: An fMRI study. Brain Research. Cognitive Brain Research, 20, 226-241.

Gelbard-Sagiv, H., Mukamel, R., Harel, M., Malach, R., \& Fried, I. (2008). Internally generated reactivation of single neurons in human hippocampus during free recall. Science, 322, 96-101.

Giacino, J. T., Ashwal, S., Childs, N., Cranford, R., Jennett, B., Katz, D. I., et al. (2002). The minimally conscious state: Definition and diagnostic criteria. Neurology, 58, 349-353.

Giacino, J. T., Hirsch, J., Schiff, N., \& Laureys, S. (2006). Functional neuroimaging applications for assessment and rehabilitation planning in patients with disorders of consciousness. Archives of Physical Medicine and Rehabilitation, 87, S67-S76.

Giacino, J. T., Kalmar, K., \& Whyte, J. (2004). The JFK coma recovery scale-revised: Measurement characteristics and diagnostic utility. Archives of Physical Medicine and Rehabilitation, 85, 2020-2029.

Gilbert, S. J., Dumontheil, I., Simons, J. S., Frith, C. D., \& Burgess, P. W. (2007). Comment on "Wandering minds: The default network and stimulus-independent thought". Science, 317, 43. author reply 43.

Goldberg, I., Ullman, S., \& Malach, R. (2008). Neuronal correlates of "free will" are associated with regional specialization in the human intrinsic/default network. Consciousness and Cognition, 17, 587-601.

Goldberg, I. I., Harel, M., \& Malach, R. (2006). When the brain loses its self: Prefrontal inactivation during sensorimotor processing. Neuron, 50, 329-339.

Golland, Y., Bentin, S., Gelbard, H., Benjamini, Y., Heller, R., Nir, Y., et al. (2007). Extrinsic and intrinsic systems in the posterior cortex of the human brain revealed during natural sensory stimulation. Cerebral Cortex, 17, 766-777.

Golland, Y., Golland, P., Bentin, S., \& Malach, R. (2008). Data-driven clustering reveals a fundamental subdivision of the human cortex into two global systems. Neuropsychologia, 46, 540-553.

Greicius, M. D., Krasnow, B., Reiss, A. L., \& Menon, V. (2003). Functional connectivity in the resting brain: A network analysis of the default mode hypothesis. Proceedings of the National Academy of Sciences of the United States of America, 100, 253-258.

Greicius, M. D., Srivastava, G., Reiss, A. L., \& Menon, V. (2004). Default-mode network activity distinguishes Alzheimer's disease from healthy aging: Evidence from functional MRI. Proceedings of the National Academy of Sciences of the United States of America, 101, 4637-4642.

Gusnard, D. A., Raichle, M. E., \& Raichle, M. E. (2001). Searching for a baseline: Functional imaging and the resting human brain. Nature reviews. Neuroscience, 2, 685-694. 
Hagmann, P., Cammoun, L., Gigandet, X., Meuli, R., Honey, C. J., Wedeen, V. J., et al. (2008). Mapping the structural core of human cerebral cortex. PLoS Biology, 6, e159.

Hasson, U., Nir, Y., Levy, I., Fuhrmann, G., \& Malach, R. (2004). Intersubject synchronization of cortical activity during natural vision. Science, 303, 1634-1640.

He, B. J., Snyder, A. Z., Zempel, J. M., Smyth, M. D., \& Raichle, M. E. (2008). Electrophysiological correlates of the brain's intrinsic large-scale functional architecture. Proceedings of the National Academy of Sciences of the United States of America, 105, 16039-16044.

Hirsch, J. (2005). Functional neuroimaging during altered states of consciousness: How and what do we measure? Progress in Brain Research, 150, 25-43.

Honey, C. J., Sporns, O., Cammoun, L., Gigandet, X., Thiran, J. P., Meuli, R., et al. (2009). Predicting human resting-state functional connectivity from structural connectivity. Proceedings of the National Academy of Sciences of the United States of America, 106, 2035-2040.

Hyvärinen, A. (1999). Fast and robust fixed-point algorithms for independent component analysis. IEEE Transactions on Neural Networks, 10(3), 626-634.

Jennett, B., \& Plum, F. (1972). Persistent vegetative after brain damage. Lancet, $1,4$.

Laureys, S. (2005). The neural correlate of (un)awareness: Lessons from the vegetative state. Trends in Cognitive Sciences, 9, 556-559.

Laureys, S., Faymonville, M. E., Degueldre, C., Fiore, G. D., Damas, P., Lambermont, B., et al. (2000). Auditory processing in the vegetative state. Brain, 123(Pt 8), 1589-1601.

Laureys, S., Giacino, J. T., Schiff, N. D., Schabus, M., \& Owen, A. M. (2006). How should functional imaging of patients with disorders of consciousness contribute to their clinical rehabilitation needs? Current Opinion in Neurology, 19, 520-527.

Laureys, S., Goldman, S., Phillips, C., Van Bogaert, P., Aerts, J., Luxen, A., et al. (1999a). Impaired effective cortical connectivity in vegetative state: Preliminary investigation using PET. Neuroimage, 9, 377-382.

Laureys, S., Lemaire, C., Maquet, P., Phillips, C., \& Franck, G. (1999b). Cerebral metabolism during vegetative state and after recovery to consciousness. Journal of Neurology, Neurosurgery, and Psychiatry, 67, 121.

Laureys, S., Owen, A. M., \& Schiff, N. D. (2004). Brain function in coma, vegetative state, and related disorders. Lancet Neurology, 3, 537-546.

Laureys, S., Perrin, F., \& Brédart, S. (2007). Self-consciousness in non-communicative patients. Consciousness \& Cognition, 16, 722-741.

Lowe, M. J., Mock, B. J., \& Sorenson, J. A. (1998). Functional connectivity in single and multislice echoplanar imaging using resting-state fluctuations. Neuroimage, 7, 119-132.

Majerus, S., Gill-Thwaites, H., Andrews, K., \& Laureys, S. (2005). Behavioral evaluation of consciousness in severe brain damage. Progress in Brain Research, 150, 397-413.

Mantini, D., Perrucci, M. G., Del Gratta, C., Romani, G. L., \& Corbetta, M. (2007). Electrophysiological signatures of resting state networks in the human brain. Proceedings of the National Academy of Sciences of the United States of America, 104, 13170-13175.

Mason, M. F., Norton, M. I., Van Horn, J. D., Wegner, D. M., Grafton, S. T., \& Macrae, C. N. (2007). Wandering minds: The default network and stimulus-independent thought. Science, 315, 393-395.

McKeown, M. J., Makeig, S., Brown, G. G., Jung, T. P., Kindermann, S. S., Bell, A. J., et al. (1998). Analysis of fMRI data by blind separation into independent spatial components. Human Brain Mapping, 6, 160-188.

Mitra, P. P., Ogawa, S., Hu, X. P., \& Ugurbil, K. (1997). The nature of spatiotemporal changes in cerebral hemodynamics as manifested in functional magnetic resonance imaging. Magnetic Resonance in Medicine, 37(4), 511-518.

Naccache, L., Obadia, M., Crozier, S., Detante, O., Guillerm, C., Bonneville, F., et al. (2004). Preserved auditory cognitive ERPs in severe akinetic mutism: A case report. Brain Research. Cognitive Brain Research, 19, 202-205.

Nir, Y., Hasson, U., Levy, I., Yeshurun, Y., \& Malach, R. (2006). Widespread functional connectivity and fMRI fluctuations in human visual cortex in the absence of visual stimulation. Neuroimage, 30, 1313-1324.

Nir, Y., Mukamel, R., Dinstein, I., Privman, E., Harel, M., Fisch, L., et al. (2008). Interhemispheric correlations of slow spontaneous neuronal fluctuations revealed in human sensory cortex. Nature Neuroscience, 11, 1100-1108.

Owen, A. M., Coleman, M. R., Boly, M., Davis, M. H., Laureys, S., \& Pickard, J. D. (2006). Detecting awareness in the vegetative state. Science, 313, 1402.

Owen, A. M., Coleman, M. R., Boly, M., Davis, M. H., Laureys, S., \& Pickard, J. D. (2007). Using functional magnetic resonance imaging to detect covert awareness in the vegetative state. Archives of Neurology, 64, 1098-1102.

Perlbarg, V., Bellec, P., Anton, J. L., Pelegrini-Issac, M., Doyon, J., \& Benali, H. (2007). CORSICA: Correction of structured noise in fMRI by automatic identification of ICA components. Magnetic Resonance Imaging, 25, 35-46.

Perlbarg, V., \& Marrelec, G. (2008). Contribution of exploratory methods to the investigation of extended large-scale brain networks in functional MRI: Methodologies, results, and challenges. International Journal of Biomedical Imaging, 2008, 218519. doi:10.1155/2008/218519.

Plum, F., \& Posner, J. B. (1972). The diagnosis of stupor and coma. Contemporary Neurology Series, 10, 1-286.

Raichle, M. E., MacLeod, A. M., Snyder, A. Z., Powers, W. J., Gusnard, D. A., \& Shulman, G. L. (2001). A default mode of brain function. Proceedings of the National Academy of Sciences of the United States of America, 98, 676-682.

Raichle, M. E., \& Snyder, A. Z. (2007). A default mode of brain function: A brief history of an evolving idea. Neuroimage, 37, 1083-1090. Discussion 1097-9.

Rombouts, S. A., Damoiseaux, J. S., Goekoop, R., Barkhof, F., Scheltens, P., Smith, S. M., et al. (2009). Model-free group analysis shows altered BOLD FMRI networks in dementia. Human Brain Mapping, 30, 256-266. 
Schiff, N. D. (2006a). Measurements and models of cerebral function in the severely injured brain. Journal of Neurotrauma, 23, 1436-1449.

Schiff, N. D. (2006b). Multimodal neuroimaging approaches to disorders of consciousness. The Journal of Head Trauma Rehabilitation, 21, 388-397.

Schiff, N. D., Rodriguez-Moreno, D., Kamal, A., Kim, K. H., Giacino, J. T., Plum, F., et al. (2005). fMRI reveals largescale network activation in minimally conscious patients. Neurology, 64, 514-523.

Schnakers, C., Perrin, F., Schabus, M., Hustinx, R., Majerus, S., Moonen, G., et al. (2009). Detecting consciousness in a total locked-in syndrome: An active event related paradigm. Neurocase, 25, 1-7.

Seeley, W. W., Crawford, R. K., Zhou, J., Miller, B. L., \& Greicius, M. D. (2009). Neurodegenerative diseases target large-scale human brain networks. Neuron, 62, 42-52.

Smart, C. M., Giacino, J. T., Cullen, T., Moreno, D. R., Hirsch, J., Schiff, N. D., et al. (2008). A case of locked-in syndrome complicated by central deafness. Nature Clinical Practice. Neurology, 4, 448-453.

Sorger, B., Dahmen, B., Reithler, J., Gosseries, O., Maudoux, A., Laureys, S., Goebel, R. (2009). Another kind of 'BOLD response': Answering multiple-choice questions via online decoded single-trial brain signals, Progress in Brain Research (this volume).

Tian, L., Jiang, T., Liu, Y., Yu, C., Wang, K., Zhou, Y., et al. (2007). The relationship within and between the extrinsic and intrinsic systems indicated by resting state correlational patterns of sensory cortices. Neuroimage, 36, 684-690.

Vanhaudenhuyse, A., Noirhomme, Q., Tshibanda, J.-F. L., Bruno, M.-A., Boveroux, P., Schnakers, C., et al. (Submitted). Default network connectivity reflects the level of consciousness in non-communicative brain damaged patients. Brain.

Vincent, J. L., Patel, G. H., Fox, M. D., Snyder, A. Z., Baker, J. T., Van Essen, D. C., et al. (2007). Intrinsic functional architecture in the anaesthetized monkey brain. Nature, 447, 83-86.

Wang, K., Yu, C., Xu, L., Qin, W., Li, K., Xu, L., et al. (2009). Offline memory reprocessing: Involvement of the brain's default network in spontaneous thought processes. PLoS ONE, 4, e4867.

Xiong, J., Parsons, L. M., Gao, J. H., \& Fox, P. T. (1999). Interregional connectivity to primary motor cortex revealed using MRI resting state images. Human Brain Mapping, 8, 151-156. 